talc after intrapleural administration: an experimental study in rats. Chest 2002; 122: 1737-1741.

10 Janssen JP, Collier G, Astoul P, et al. Safety of pleurodesis with talc poudrage in malignant pleural effusion: a prospective cohort study. Lancet 2007; 369: 1535-1539.

11 Weissberg D, Refaely Y. Pneumothorax. Experience with 1,199 patients. Chest 2000; 117: 1279-1285.
12 Cardillo G, Carleo F, Giunti R, et al. Videothoracoscopic talc poudrage in primary spontaneous pneumothorax: a single-institution experience in 861 cases. J Thorac Cardiovasc Surg 2006; 131: 322-328.

\title{
Methodological issues in therapeutic trials of COPD
}

\section{To the Editors:}

I read with great interest the paper by SuISSA et al. [1], which has shed some light on the pitfalls when evaluating the effects of inhaled corticosteroids (ICS) on chronic obstructive pulmonary disease exacerbations. Indeed, they conclude, "various methodological shortcomings ...the effectiveness of inhaled corticosteroids in treating chronic obstructive pulmonary disease remains doubtful" [1]. The controversy about the role of ICS in COPD exacerbations is becoming even more complex, with the VIVACE [2] study reporting that pneumonia is associated with ICS use in COPD, a possible effect also reported in several more recent studies [3-5]. Moreover, the considerable cost of ICS treatment should also be taken into account. Several other issues have been raised AARON et al. [6] and some, but not all, of the aspects were already reflected [7].

The observed differences between former ICS users and ICS naïve COPD patients in the OPTIMAL trial [1] is impressive but may not be generalised. In other trials this effect is much less remarkable and does not reach clinical relevance. In a post hoc analysis we have examined the ICS effect on exacerbations in former ICS, long-acting $\beta$-agonists and combination product users and nonusers in the VIVACE study [2]. VIVACE was a 1yr trial in which salmeterol was compared with salmeterol/ fluticasone. Irrespective of former ICS use, we observed that patients benefited from additional ICS treatment compared with salmeterol alone. Indeed, the benefit in former ICS users appeared greater (table 1). However, our post hoc analysis shows that the ICS effect in VIVACE is exclusively attributable neither to current bronchodilator therapy nor to former ICS use. Thus the common clinical practice of initiating ICS treatment in COPD patients with frequent exacerbations remains advisable.

Time to first exacerbation was analysed by the Cox proportional hazard model with the following predictive variables: treatment; sex; age; smoking status; forced expiratory volume in one second ( $\%$ predicted at the start of randomised treatment period); and long-acting $\beta$-adrenergic use pre-study. The frequency of exacerbations was analysed using a Poisson regression model with the same covariables as for time to first exacerbation.

The classical intention to treat analysis is helpful in assessing, conservatively, the effect of an intervention and also prevents bias from the regression artifact particularly in a study population with differential (not at random) dropout rates. Conversely, however, from a clinical point of view, in a 3-yr pharmacological trial such as TORCH [3] one expects to measure the effect of a current (continuing) treatment on an outcome. An intention-to-treat analysis will assume that, at the

TABLE 1 Treatment effect (salmeterol relative to salmeterol/fluticasone) according to pre-trial inhaled corticosteroid (ICS) use

HR: hazard ratio; Cl: confidence interval; RR: ratio rate. Values more than one indicate a higher rate for salmeterol relative to salmeterol/fluticasone treatment. Time to first exacerbation was analysed by Cox's proportional hazard model with the following predictive variables: treatment, sex, age, smoking status, forced expiratory volume in one second (\% predicted at start of randomised treatment period) and long-acting $\beta$-adrenergic use pre-study. Count of exacerbation was analysed by use of a Poisson regression model with the same covariables as for time to first exacerbation. 
end of the study, the treatment has efficacy even if 1 or 2 yrs have elapsed since the patient stops treatment. To date, there is no convincing evidence to support such an assumption. Unknown usual care treatment for withdrawals including ICS could further bias the results. Thus, for short-term secondary outcomes (e.g. exacerbations) the intention-to-treat analysis might not produce robust and meaningful results. Estimating and correcting for regression towards the mean could help in achieving clinically more reliable results.

In addition, AARON et al. [6] argue elsewhere that premature termination of a drug, for whatever reason (including withdrawal of informed consent), might indicate both impaired tolerance and lack of efficacy. Thus, regarding the end-point withdrawal as just another measure of effectiveness. Effectiveness, however, is not the efficacy of a drug in a randomised controlled trial, but in the real life medical care situation, which may be quite different.

Therefore the correct statistical methodology might be complex and would require many variables to be considered: the population studied; duration of the study; data on recent and subsequent treatment in patients who discontinued prematurely; characteristics of the treatment; and properties of the outcome variable.

In conclusion, as a clinician I agree with SuISSA et al. [1] that rigorous study design and conservative assessment are urgently needed to avoid spurious results when studying drug treatment in chronic obstructive pulmonary disease. Particularly in exacerbation trials the bias that might arise from inhaled corticosteroids pre-treatment and withdrawal should be accounted for (even if we assume it does not necessarily confuse the effect in every case). The continued evolution of statistics and our knowledge of the complex disease chronic obstructive pulmonary disease might allow better clinical and statistical assessment of the population, the drug under investigation, the treatment effects and withdrawals in each study. So far it appears no "one size fits all" method is readily available.

\section{P. Kardos}

Group Practice and Centre for Respiratory Medicine, Allergy and Somnology at Maingau Hospital, Frankfurt am Main, Germany.

\section{STATEMENT OF INTEREST}

A statement of interest for P. Kardos can be found at www.erj.ersjournals.com/misc/statements.shtml

\section{REFERENCES}

1 Suissa S, Ernst P, Vandemheen KL, Aaron SD. Methodological issues in therapeutic trials of COPD. Eur Respir J 2008; 31: 927-933.

2 Kardos P, Wencker M, Glaab T, Vogelmeier C. Impact of salmeterol/fluticasone propionate versus salmeterol on exacerbations in severe chronic obstructive pulmonary disease. Am J Respir Crit Care Med 2007; 175: 144-149.

3 Calverley PM, Anderson JA, Celli B, et al. Salmeterol and fluticasone propionate and survival in chronic obstructive pulmonary disease. N Engl J Med 2007; 356: 775-789.

4 Wedzicha JA, Calverley PMA, Seemungal TA, et al. The prevention of chronic obstructive pulmonary disease exacerbations by salmeterol/fluticasone propionate or tiotropium bromide. Am J Respir Crit Care Med 2008; 177: 19-26.

5 Ernst P, Gonzalez AV, Brassard P, Suissa S. Inhaled corticosteroid use in COPD and the risk of hospitalization for pneumonia. Am J Respir Crit Care Med 2007; 176: 162-166.

6 Aaron SD, Fergusson D, Marks GB, et al. Counting, analysing and reporting exacerbations of COPD in randomised controlled trials. Thorax 2008; 63: 122-128.

7 Cooper CB, Decramer M. Inhaled steroids and COPD. Eur Respir J 2008; 32: 523-524.

\section{Inhaled insulin does not trigger lung inflammation and airway remodelling}

\section{To the Editors:}

We read with great interest the paper by LIU et al. [1], regarding the effects of inhaled insulin on airway lining fluid composition in adults with diabetes. The study concluded that treatment with inhaled human insulin (Exubera ${ }^{\circledR}$ ) is not associated with evidence of pulmonary inflammation and therefore the treatment effects on lung function observed in Exubera ${ }^{\circledR}$ trials are not caused by lung inflammation.

Local inflammation results in airway remodelling mediated by cytokines produced by recruited inflammatory cells, as well as by airway myofibroblasts and airway smooth muscle (ASM) cells [2]. ASM cells maintain their plasticity exhibiting a differentiated contractile or a synthetic proliferative phenotype in response to physiological and pathological signals [3]. In a relevant study, we examined the possibility that growthpromoting properties of inhaled insulin play a role in airway remodelling [4]. Our findings regarding insulin's effect on rabbit tracheal ASM cell proliferation, show that insulin transiently promotes cell proliferation in ASM cells by the activation of the phosphoinositide 3-kinases. Importantly, longer incubation with insulin didn't lead to additional cell growth.

Therefore, the in vivo study by LiU et al. [1] together with our in vitro study [4] suggest that long-term exposure of the airways to insulin, as in the administration of inhaled insulin in 